\title{
26.3: Multidirectional Asymmetrical Microlens-Array Light Control Films for High Performance Reflective Liquid Crystal Displays
}

\author{
Yi-Pai Huang, Fu-Jen Ko*, and Han-Ping David Shieh
}

Institute of Electro-Optical Engineering, National Chiao Tung University, Hsinchu, Taiwan, 30010, R.O.C

*Present address: AU Optronics Corp., Hsinchu, Taiwan, 30010, R.O.C

\author{
Juie-Jun Chen
}

Wintek Corp., Taichung, Taiwan, 42701, R.O.C

Shin-Tson Wu

School of Optics/CREOL, University of Central Florida, Orlando, FL 32816

\begin{abstract}
The multidirectional asymmetrical microlens array light control film (MAMA-LCF) is developed to enhance the image brightness and contrast ratio of reflective liquid crystal displays. Through optimized designs and optical alignments, the MAMA-LCF which is constructed with asymmetrical microlens arrays, leads to a $\sim 5 x$ gain in brightness over the $\mathrm{MgO}$ standard white and 12:1 contrast ratio for color STN-LCDs, 10x gain and 11.5:1 contrast ratio for $P D L C$, and $9 x$ gain over the conventional Ch-LCD. In each display, the light control film does not induce visible surface diffusion, moiré patterns and parallax.
\end{abstract}

\section{Introduction}

Reflective liquid crystal displays (LCDs) ${ }^{1}$ are being widely used in portable personal digital assistants and mobile communications. Varieties of new applications, e.g., STN-LCDs for mobile phones, PDLC for smart cards, and cholesteric LCD for e-books have been considered. In such applications, low power consumption, high brightness, high contrast ratio, and low cost are critical. Most single polarizer-based reflective color LCDs still suffer from inadequate reflectivity and contrast ratio (CR).

Many methods, such as rough surface of reflector, ${ }^{2}$ holographic reflector, ${ }^{3}$ front scattering film, ${ }^{4}$ holographic light control film, ${ }^{5,6}$ and asymmetrical microlens array light control film, ${ }^{7}$ have been developed for improving the brightness and contrast ratio of reflective LCDs. We have developed a modified multidirectional asymmetrical microlens array light control film (MAMA-LCF) ${ }^{8}$ by including multiple ambient illuminations. As a result, the viewing angle is widened and reflectance enhanced. Simple fabrication and low cost are the major advantages of the MAMA light control film. In this paper, we demonstrate the performances of MAMA light control film on three reflective LCDs: color STN, PDLC and Ch-LCD. Through optimized designs and alignments, the image quality of these displays is improved significantly.

\section{Reflective LCDs with MAMA-LCF}

\subsection{Reflective Color STN}

The design patterns and system configuration of the MAMA-LCF on reflective color STN-LCDs are shown schematically in Figs. 1(a) and (b), respectively. In a reflective color STN-LCD, the

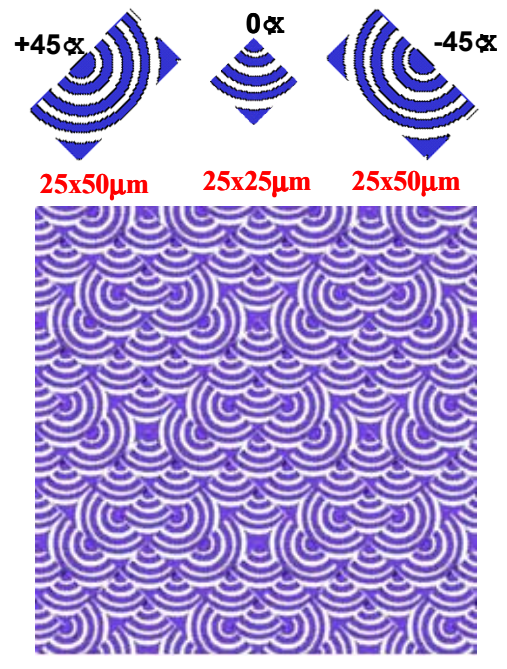

(a)

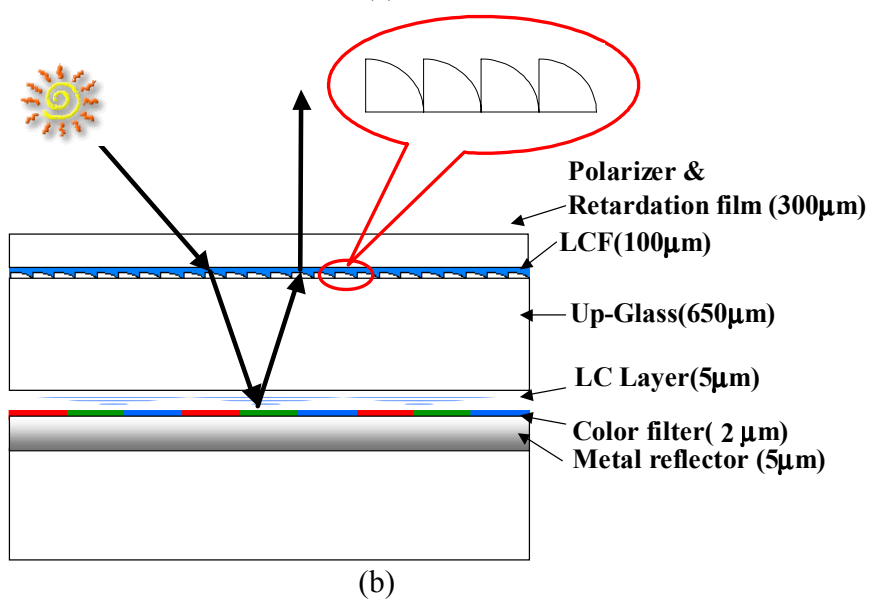

Fig. 1. (a) The design pattern and (b) panel configuration of a reflective color STN using a MAMA-LCF.

color pixel size is $210 \mu \mathrm{m} \times 210 \mu \mathrm{m}$ which covers more than 50 microlenses, as shown in Fig. 1(a). Thus, the moiré patterns are not visible because the designed structure and the pitch of the microlenses are much smaller than the pixel size. Moreover, the 
MAMA-LCF is made of low birefringence material and laminated between the polarizer and top-glass, as shown in Fig. 1(b). As a result, the surface diffusion is low and parallax remains invisible.

\subsection{PDLC}

MAMA-LCF is also applicable to the reflective PDLC which has been developed for plastic smart cards. Usually, the MAMA-LCF is laminated on the top surface of PDLC. Under such circumstance, the film would modulate the reflected light from the interface of each layer, and deteriorate the blackness of the dark state, as shown in the left part of Fig. 2(b). Thus, the MAMA-LCF is preferred to be laminated between the bottom substrate of the plastic PDLC panel and the aluminum reflector, as shown in Fig. 2(a). The dark state photograph of the bottom-aligned structure is shown in the right side of Fig. 2(b). From Fig. 2, the bottomlaminated MAMA light control film shows a much darker state than that on the top. Moreover, the plastic LCF is flexible and can be easily combined with the plastic displays.

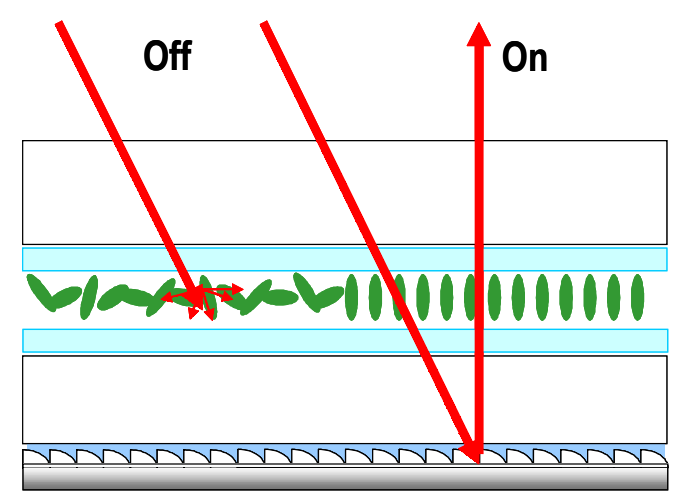

PET

ITO

PDLC

ITO

PET

LCF

Al Reflector

\section{LCF on Bottom}

(a)

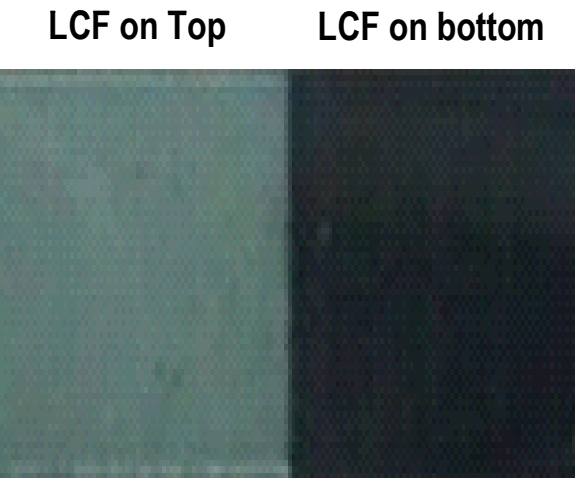

Dark State

(b)

Fig. 2. (a) Aligned structure of MAMA-LCF with PDLC displays. (b) Photograph of the dark state of a PDLC with MAMA-LCF on top (left) and bottom (right).

\subsection{Cholesteric LCD}

Cholesteric liquid crystal display (Ch-LCD) $)^{9,10}$ is a strong contender for electronic-books because of its low power consumption. In a conventional Ch-LCD, in order to achieve wide viewing angle, only the top substrate is rubbed and the bottom plate has no rubbing. The LC directors tilt to different angles, as sketched in Fig. 3(a). These slightly disordered cholesteric layers help diffuse the reflected light to a wider viewing zone. The tradeoff of this approach is that the maximum reflectivity is reduced to $35 \%$. On the other hand, the two-surface rubbed cell exhibits a higher $(\sim 50 \%)$ reflectivity except that its viewing angle is much narrower. Our motivation is to integrate a MAMA light control film on the two-surface rubbed cell for widening viewing angle while preserving high reflectivity. As shown in Fig. 3(b), the MAMA light control film helps to diffuse light to a larger angle and reduces surface reflections in the viewing zone. Benefiting from the light control film, the two-surface rubbed Ch-LCD is expected to exhibit a bright image and wide viewing angle.

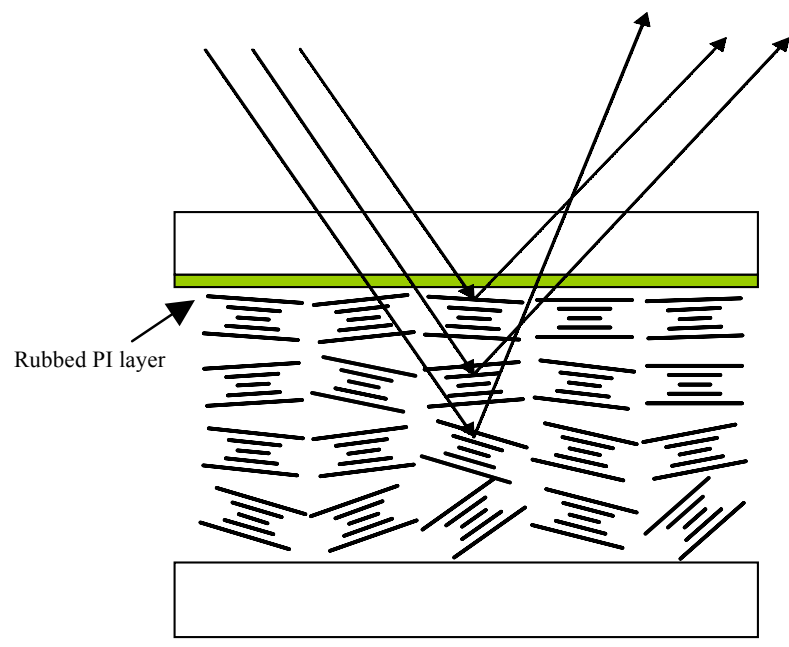

(a)

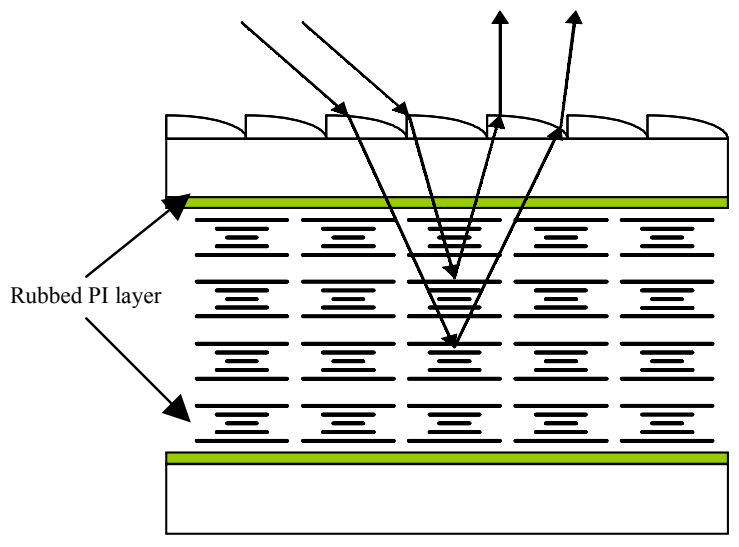

(b)

Fig. 3 Schematic drawings of (a) one-surface and (b) two-surface rubbed Ch-LCD with MAMA-LCF. 


\section{Experiment Results}

The angular-dependent reflectivity and CR of a color STN and a PDLC cells are measured and results are shown in Figs. 4-5, respectively. For a collimated illumination from $-30^{\circ}$, the specular reflection occurs at $30^{\circ}$. At this angle, although the reflectivity is high, the contrast ratio is poor. Adding a MAMA light control film not only shifts the peak reflectance of the STN panel from $30^{\circ}$ to $14^{0}$ but also enhances reflectivity by $\sim 5 \mathrm{x}$ over the $\mathrm{MgO}$ standard white, as shown by the solid line in Fig. 4(a). Similarly, the reflectance profile of a PDLC with and without MAMA-LCF shown in Fig. 4(b) also proves that MAMA-LCF yields a much higher brightness $(\sim 10 \mathrm{x}$ of $\mathrm{MgO})$. The contrast ratios of the color STN and PDLC with MAMA-LCF are shown by the solid lines in Figs. 5(a) and (b), respectively. The maximum CR 12:1 is higher than those of the commonly used diffuser and bump reflector.

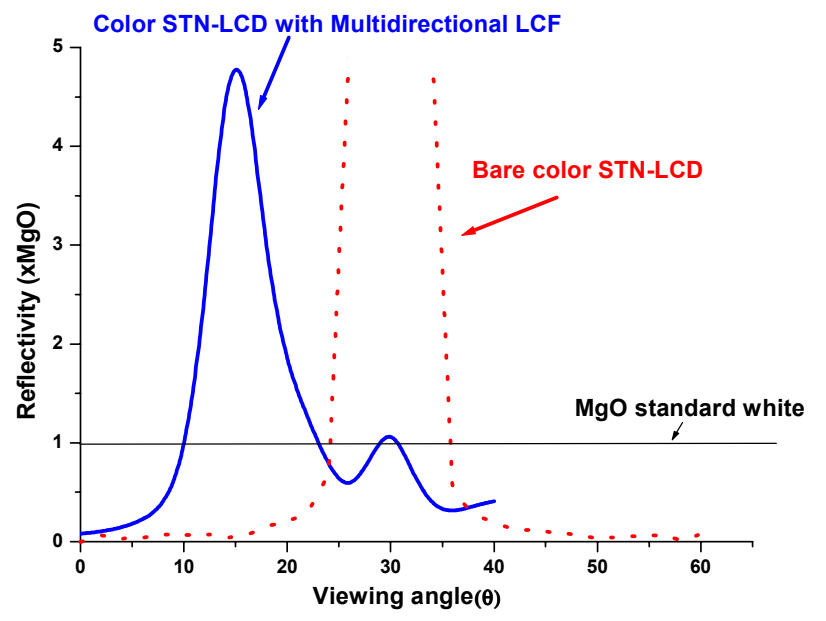

(a)

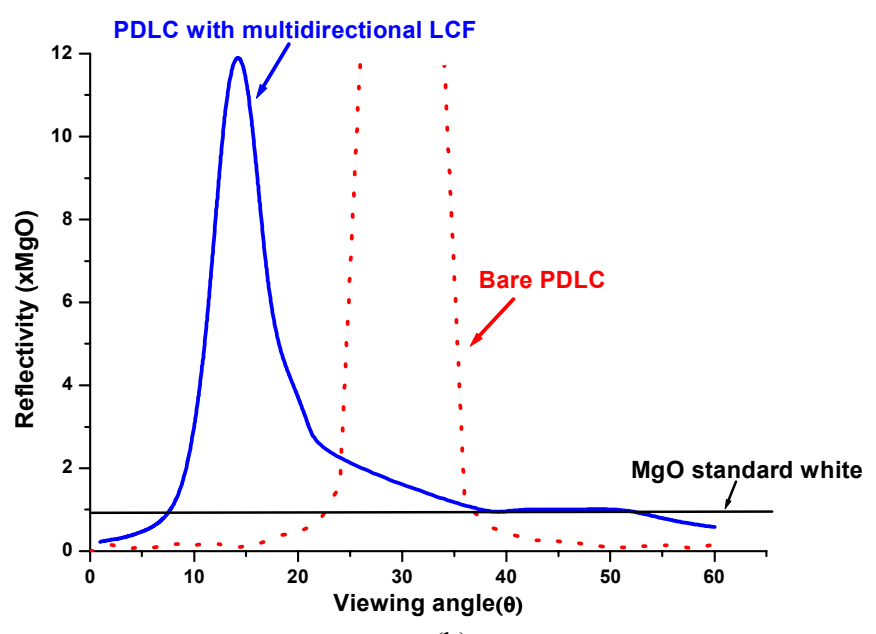

(b)

Fig. 4. The measured reflectivity of (a) color STN and (b) PDLC as a function of viewing angle under illumination from $-30^{\circ}$.

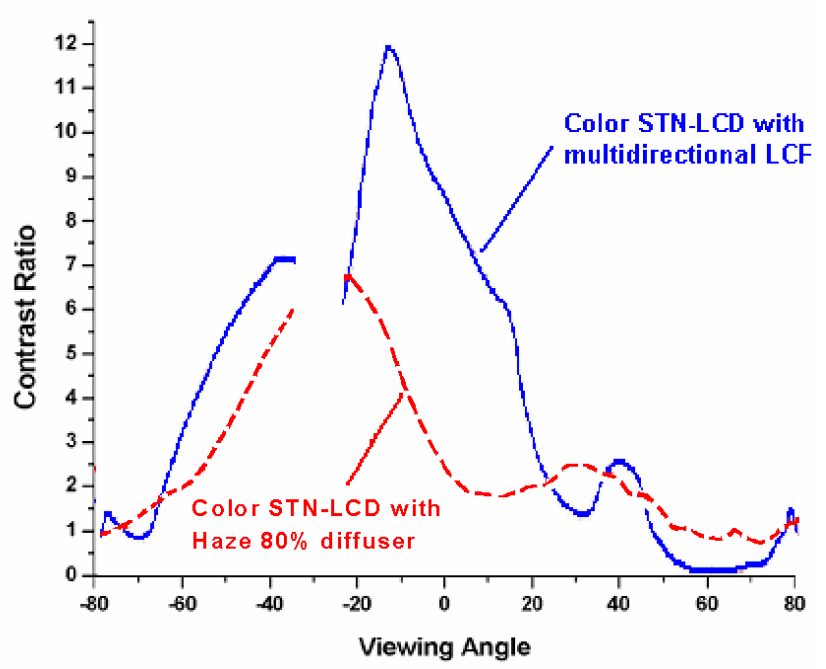

(a)

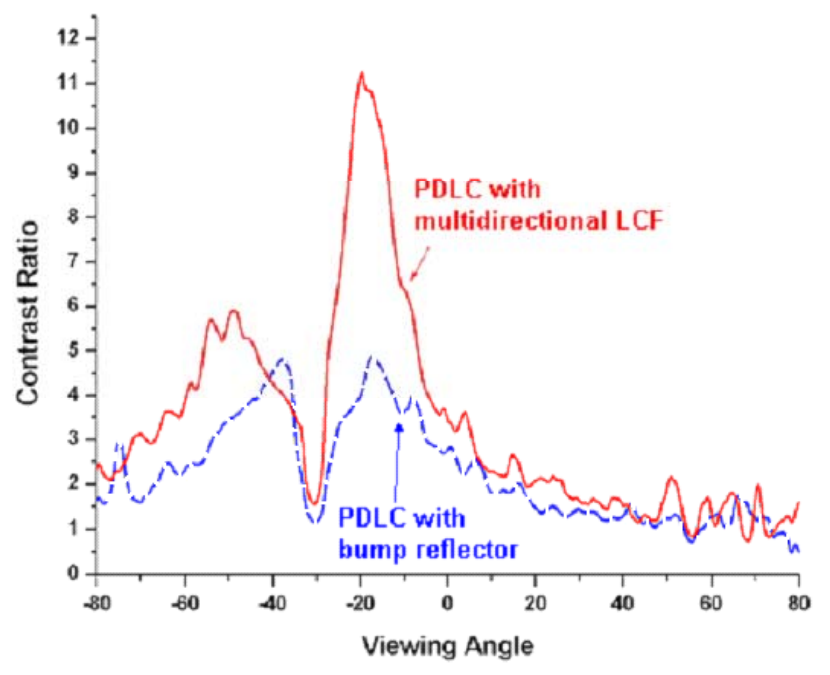

(b)

Fig. 5. The measured CR of (a) color STN and (b) PDLC as a function of viewing angle under illumination from $-30^{\circ}$.

For the Ch-LCD experiments, we have prepared two cells using E48 LC doped with ZLI-811 chiral agent. The peak wavelength is at green. The back side of the cell was painted black for improving contrast ratio. A green diode laser was used to illuminate the $\mathrm{Ch}$ LCD cells at $-30^{\circ}$. The MAMA light control film was laminated on the top surface of the Ch-LCD cell. Fig. 6 plots the measured reflectance ratio (at $\mathrm{V}=0$ ) of the two-surface buffed Ch-LCD with a MAMA-LCF over the one-surface buffed Ch-LCD without MAMA-LCF. From Fig. 6, the MAMA-LCF/Ch-LCD shows a higher reflectance in the $10-25^{\circ}$ viewing zone. At $18^{\circ}$, the light control film enhances the display brightness by a factor of 9 . 


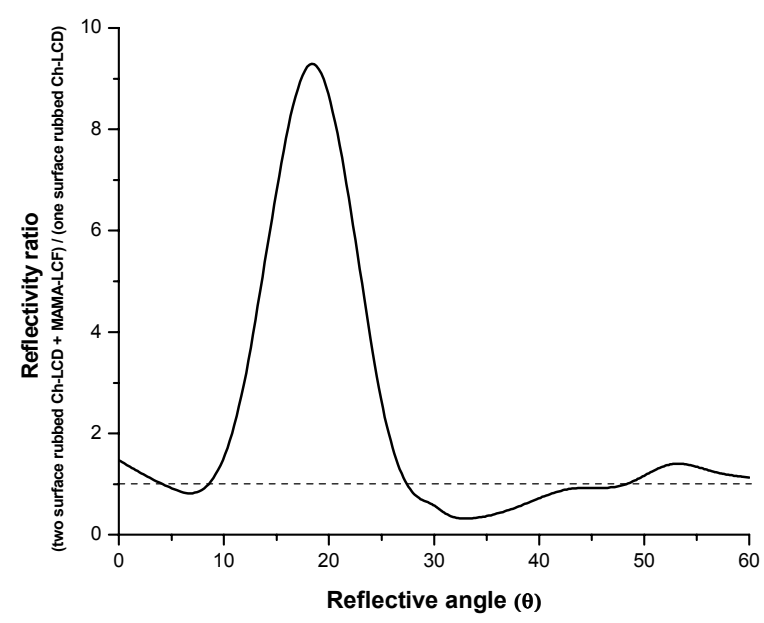

Fig. 6 Reflectance comparison of the two-surface buffed Ch-LCD with a MAMA-LCF over the top-surface buffed Ch-LCD without MAMA-LCF.

The photographs of displayed images using the MAMA-LCF on a color STN-LCD, PDLC and Ch-LCD, taken under ambient light condition are shown at the top of Figs. 7(a), (b) and (c), respectively. In comparison, the bottom of Fig. 7(a) shows bare STN-LCD (left) and STN-LCD with an $80 \%$ haze diffuser (right), which is commonly used to enhance the brightness of mobile displays. The photographs of PDLC with bump reflector and a bare PDLC are shown in the middle and bottom sections of Fig. 7(b). Additionally, the middle and bottom sections of Fig. 7(c) show the one-surface buffed Ch-LCD and the bare two-surface buffed Ch-LCD. The image quality with the MAMA light control film on the three different LCDs is clearly enhanced.

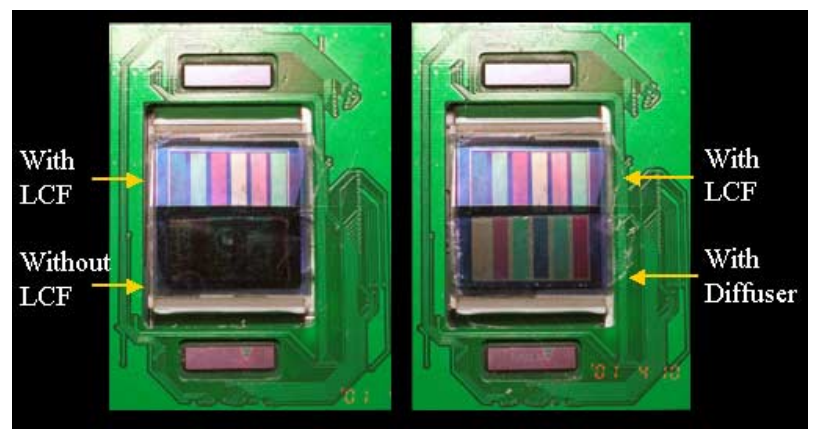

(a)

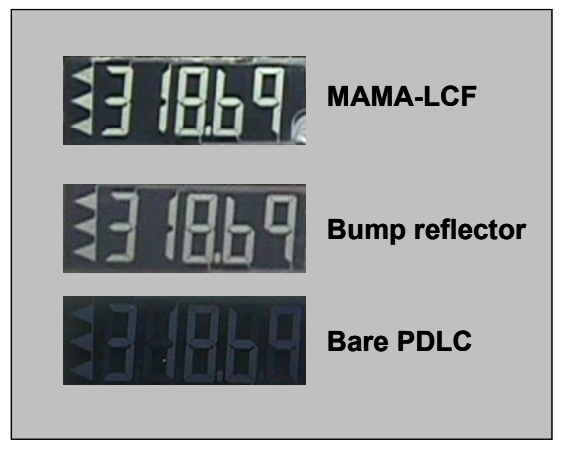

(b)

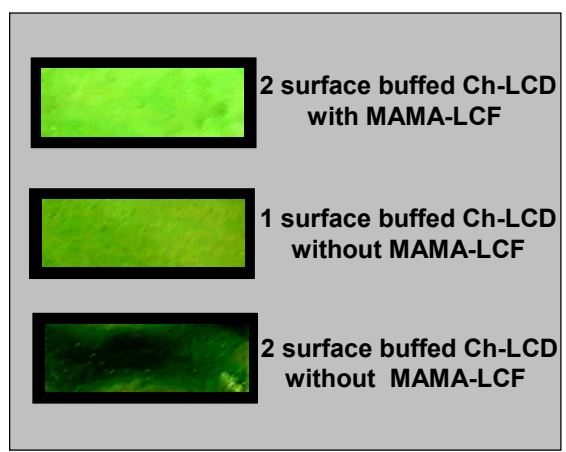

(c)

Fig.7. Sample photographs of (a) color-STN LCD, (b) PDLC, and (c) Ch-LCD. The displays with MAMA-LCF clearly show much brighter images.

\section{Conclusions}

The use of MAMA-LCF effectively enhances the display brightness and image quality of the color STN, PDLC and ChLCD under ambient light condition. The dispersion, moiré patterns, and parallax are all invisible. The MAMA light control films can be easily fabricated by standard semiconductor processes and injection/stamping molding. By using these welldeveloped fabrication processes, the designed microlens structure on thin transparent plastic substrate can be produced economically and reproducibly in large volume. The wide spread application of MAMA light control film for reflective LCDs are foreseeable.

\section{Acknowledgements}

This work was partially supported by National Science Council, the Republic of China under contract no. NSC89-2215-E009-082, Wintek Corporation and Alien technology ${ }^{\mathrm{TM}}$. We would like to express our appreciation to Dr. Fredric Vicentini of Alien technology ${ }^{\mathrm{TM}}$, Dr. Hongwen Ren of University of Central Florida, Mr. Jérôme REINEIX and Mr. Cyrus VOTA of Université Paris Sud center d'Orsay, Mr. K. H. Liu of ERSO/ITRI, Mr. Yih-Chun $\mathrm{Wu}$ and Mr. Johnny Wang of Wintek Corp. for valuable discussion and technical support.

\section{References}

1. S. T. Wu and D. K. Yang, "Reflective Liquid Crystal Displays", (Wiley-SID, 2001).

2. Y. Itoh, S. FuJiwara, N. Kimura, S. Mizushima, F. Funada and M. Hijikigawa, SID’98, p.221.

3. M. Wenyon, W. Molenti and P. Ralli, SID'97, p.691.

4. T. Uchida, T. Nakayama, T. Miyashita, and T. Ishinaba, Asia Display'95, p.599.

5. G. T. Valliath, Z. A. Coleman, J. L. Schindler, R. Polak, R. B. Akins and K. W. Jelley, SID'98, p.1139.

6. H. Seki, N. Sugiura, M. Shimizu and T. Uchida, SID'96, p.614.

7. F. J. Ko and H. P. Shieh, Jpn. J. Appl. Phys. $\underline{39}$, 2647 (2000).

8. Y. P. Huang, F. J. Ko and H. P. Shieh, SID’01, p.448.

9. D. K. Yang, L. C. Chien, and J. W. Doane, SID'92, p.759.

10. Z. J. Lu, J. L. West, X. Y. Huang, D. K. Yang, J. W. Doane, SID'95 p.172. 